\title{
A Hybrid Model of 1-D Signal Adaptive Filter Based on the Complex Use of Huang Transform and Wavelet Analysis
}

\author{
Sergii Babichev \\ Jan Evangelista Purkyně University in Ustí nad Labem, Ustí nad Labem, Czech Republic \\ E-mail: sergii.babichev@ujep.cz \\ Oleksandr Mikhalyov \\ National Metallurgical Academy of Ukraine, Dnipro, Ukraine \\ E-mail: maillich2@gmail.com
}

Received: 06 August 2018; Accepted: 25 November 2018; Published: 08 February 2019

\begin{abstract}
The paper presents the results of the research concerning the development of the hybrid model of 1-D signal adaptive filter based on the complex use of both the empirical mode decomposition and the wavelet analysis. Implementation of the proposed model involves three stages. Firstly, the initial signal is decomposed to the empirical modes by the Huang transform with allocation the components which contain the noise. Then the wavelet filtering is performed to remove the noise component. The optimal parameters of the wavelet filter are determined based on the minimal value of ratio of Shannon entropy for the filtered data and the allocated noise component and these parameters are determined depending on type of the studied component of the signal. Finally, the signal is reconstructed with the use of the processed modes. The results of the simulation with the use of the test data have shown higher effectiveness of the proposed method in comparison with standard method of the signal denoising based on wavelet analysis.
\end{abstract}

Index Terms-Denoising, Empirical mode decomposition, Huang transform, Wavelet analysis, Thresholding, Shannon entropy.

\section{INTRODUCTION}

1-D and 2-D signals denoising are one of the current problems of modern informatics. Allocation of the noise component from the studied data allows us to improve the quality of the investigated signal that improves the effectiveness of the following stage of the data processing within the framework of the current problem. A lot of denoising techniques based on different methods of the signal processing exist nowadays. The use of Kalman [1] or Wiener [2] filters allows us to smooth the signal by using the extrapolation technique in the first case and by minimizing the mean square error between the estimated random process and the desired process in the second case. However, it should be noted, that these techniques are not effective in the case of processing of nonstationary and non-linear signals with local particularities. Implementation of these techniques in these cases promotes to the loss of the large amount of useful information.

The modern techniques of the non-stationary and nonlinear signals processing are based on decomposition of the investigated signals to the components with the following processing of these components in order to remove the noise. So, the research concerning the use of fast Fourie transforms (FFT) for estimation of the anisotropic relaxation of composites and nonwovens is presented in [3]. In paper [4] the authors implemented the time-frequency analysis of pressure pulsation signal based on FFT. The frequency spectrum including frequency-domain structure and approximate frequencyscope was obtained during the simulation process. However, it should be noted, that FFT is effective in the case of stationary signals processing and features extraction from the data. In the case of more complex non-stationary and non-linear signal processing the effectiveness of the FFT technique decreases. The wavelet analysis is the alternative and the logical continuation of the FFT [5, 6]. The approximation and detail coefficients are calculated during waveletdecomposition process. The detail coefficients contain the information about the noise component in the most cases, thus these coefficients are processed to remove the noise component. Reconstruction of the denoised signal is performed with the use of both the approximation coefficients and the processed detail coefficients at levels of the wavelet decomposition from 1 to $\mathrm{N}$. The wavelet analysis technique is widely used in different area of the scientific research [7-14]. The effectiveness of this technique implementation depends on the choice of the type of the used wavelet, level of the wavelet decomposition and determination of the thresholding coefficient value for detail coefficient processing. It should be noted that an effective technique for these parameters objective determining is absent nowadays. 
To process and analyze the non-stationary and nonlinear signals in $[15,16]$ authors proposed the use of the empirical mode decomposition (EMD) and the Hilbert spectrum. This approach was named Hilbert-Huang transform method (HHT). The main concept of this method consists in decomposition of the initial signal into mutually independent intrinsic mode functions (IMFs) based on the empirical mode decomposition (EMD) [15]. Then, the Hilbert spectrum is formed by applying the Hilbert transform to the obtained IMFs. The analysis of the Hilbert spectrums for the selected IMFs allows us to obtain the detail information concerning particularities of the investigated signal. The HHT method has been implemented in various fields to process and analyze non-linear and non-stationary signals such as bloodpressure signal analysis, seismic data analysis, ECG signal analysis, at al. So, the paper [17] presents the technique to decompose the multicomponent microDoppler signals based on the complex use of HilbertHuang transform and analytical mode decomposition (HHT-AMD). The approach concerning the implementation of the HHT for detection, diagnostic and prediction of the degradation in the ball bearing was proposed in [18]. The papers [19-21] are devoted to the implementation of the HHT for analysis of the vibration signals from different objects. The paper [22] presents the results of the research concerning the use of HHT for the processing and analysing of ECG signal in order to diagnose the brain functionality abnormalities. The results of the research concerning the implementation of the HHT for the analysis of the non-stationary financial time series and the acoustic wave frequency spectrum characteristics of rock mass under blasting damage are presented in $[23,24]$.

However, it should be noted that in spite of the achievements in this subject area the problem of denoising of the non-stationary and non-linear signals has no effective solution nowadays. The solution of this problem can be achieved by complex use of the modern techniques of data processing $[25,26]$. In this paper we propose the hybrid model of non-stationary and nonlinear signals denoising based on the complex use of both the Huang transform and wavelet analysis. The optimal parameters of the wavelet filter for each of the intrinsic mode functions (IMFs) are determined based on the minimum value of the quantitative criterion, which is calculated as the ratio of Shannon entropy of the filtered data and the allocated noise component. In such a way the parameters of the wavelet filter are adapted to the type of the studied signal.

The aim of the research is the development of the hybrid model of 1D non-stationary signals adaptive filter based on the complex use of both the Huang transform and wavelet analysis.

The paper is organized in the following way. Section 2 presents the techniques of the empirical mode decomposition by the Huang transform and the basis of the discrete wavelet transform (DWT) of 1-D signals. Section 3 is devoted to development of the hybrid model of the 1-D signal adaptive filter based on the complex use of both the Huang transform and DWT. This model is presented as the structure block chart of the algorithm of the studied data processing. The results of the simulation of the test data denoising within the framework of the proposed technique are presented in section 4. The obtained results and discussion are presented in section 5 . Section 6 contains the conclusion.

\section{EMPIRICAL MODE DECOMPOSITION AND DisCRETE WAVELET TRANSFORM}

The empirical mode decomposition method (EMD) involves [15] what initial signal is a complex one and it can be decomposed into a series of the intrinsic mode functions (IMFs):

$$
y(x)=\sum_{i=1}^{n} f_{i}(x)+r_{n}(x)
$$

where $n$ is the number of the IMFs functions, $f_{i}(x)$ is the IMFs function on $i$-th level of the signal decomposition, $r_{n}(x)$ is the residual function, which represent the average trend of the initial signal. Implementation of the EMD method involves the following:

- $\quad$ the number of each IMFs function extrema and the number of zero crossing must be equal or must not differ by more than one;

- $\quad$ in any point of the IMFs function the mean value of the envelope defined by local maximums and local minimums should be zero.

The process of the signal decomposition is stopped if one of the following conditions is true:

- the residual function $r_{n}(x)$ does not contain more than 2-3 extrema points;

- the residual function $r_{n}(x)$ in whole interval of $\mathrm{x}$ change is insignificant in comparison with appropriate values of the IMFs functions.

The further analysis of the allocated IMFs functions allows us to select the modes, which contain the noise for their processing in order to remove the noise component by the use of discrete wavelet transform (DWT) [27]. The main idea of the discrete wavelet decomposition is presented in Fig.1.

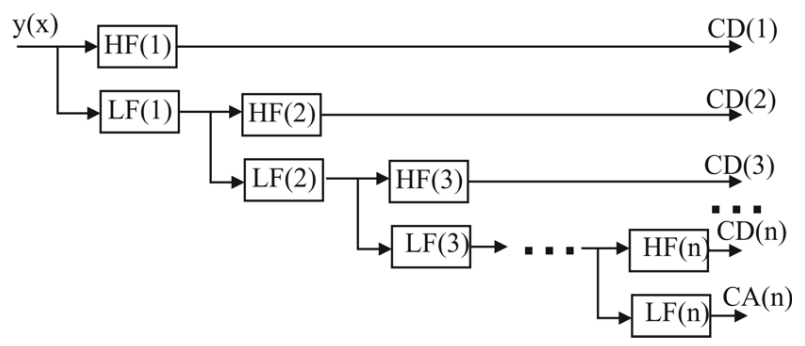

Fig.1. Structural block-chart of discrete wavelet decomposition process 
Approximation coefficients at $n$-th level and detail coefficients at the levels from 1 to $n$ are calculated with the use of both the low frequency (LF) and the high frequency (HF) filters during wavelet decomposition process:

$$
y(x) \rightarrow\{C A(n), C D(n), \ldots, C D(2), C D(1)\}
$$

The noise component in most cases is contain in detail coefficients, therefore these coefficients should be processed during the signal denoising. However, the quality of wavelet filtering depends on type of the used wavelet, wavelet decomposition level and thresholding coefficient value to process the detail coefficients. In [28] authors proposed the technology of determination of the optimal parameters of the wavelet filter based on the use of the Shannon entropy as the main criterion to select the optimal solution. The optimal wavelet and the level of wavelet decomposition are determined based on the maximum value of the Shannon entropy for the allocated noise component and the thresholding coefficient optimal value is determined based on the minimum value of the Shannon entropy for filtered data within the framework of the proposed technology. The proposed by the authors technology was successfully implemented for the gene expression profiles filtering within the framework of the information technology of gene expression profiles processing for the purpose of gene regulatory networks reconstruction [29,30]. In this paper we propose the model of the wavelet filter optimal parameters determination based on the use of ratio of the Shannon entropies for the filtered signal and the allocated noise component. The soft thresholding is used to process the detail coefficients within the framework of the proposed model:

$$
\left\{\begin{array}{l}
d=0, \text { if } d \leq \tau ; \\
d=d-\tau, \text { if } d>\tau
\end{array}\right.
$$

where $d$ are the detail coefficients and $\tau$ is the value of the thresholding coefficient. The structure block-chart of this model is presented in Fig.2.

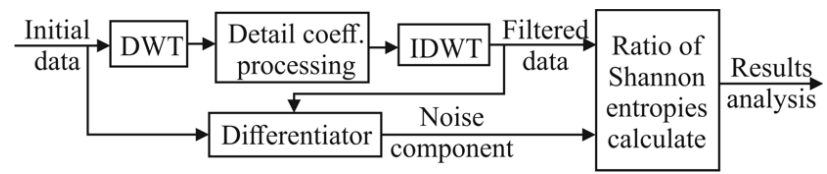

Fig.2. A model to determine the wavelet filter optimal parameters

Implementation of this model involves the following steps:

1. Fixation of the wavelet decomposition level randomly.

2. Calculation of the median absolute deviation (MAD) for the detail coefficient at the wavelet decomposition levels from 1 to $\mathrm{n}$ :

$$
\sigma=\operatorname{median}(\mid C D(i)-\text { median }(C D(i)) \mid)
$$

where $i=1, \ldots, n$ is the level of wavelet decomposition.

3. Calculation of the thresholding parameter initial value:

$$
\tau_{0}=0.1 \sigma \sqrt{2 \ln k}
$$

where $k$ is the length of the studied vector. Coefficient 0.1 was determined empirically.

4. Choice of the type of mother wavelet. Formation of the vector of wavelets which correspond to the mother wavelet.

5. Signal denoising within the range of the wavelets change by the detail coefficient processing with the following reconstruction of the signal. Selection of the noise component at each step of this procedure implementation.

6. Calculation of Shannon entropy for both the filtered data and the allocated noise component. Calculation of the ratio of the obtained criteria:

$$
R=\frac{H(\text { filtered signal })}{H(\text { noise component })} .
$$

7. Analysis of the obtained results. Choice the optimal wavelet which corresponds to the minimum value of the criterion (6).

8. Repetition of the steps from 5 to 7 of this procedure within the range of the wavelet decomposition level change from 3 to the maximum level of the wavelet decomposition with the use of the optimal wavelet. Determination of the wavelet decomposition optimal level which corresponds to the minimum value of the criterion (6).

9. Setup of both the range and the step of the thresholding parameter value change: $\tau_{\min }=0.1 \tau_{0}$; $\tau_{\max }=5 \tau_{0} ; d \tau=0.02 \cdot\left(\tau_{\max }-\tau_{\min }\right)$. Repetition of the steps from 5 to 7 of this procedure within the range of the thresholding parameter value change.

10. Determination of the thresholding parameter optimal value, which corresponds to the first minimum of the criterion (6) during enumeration of the thresholding parameter from minimum to maximum values.

11. The signal denoising with the use of the wavelet filter optimal parameters.

It should be noted, that direct implementation of the proposed technology for the denoise of the signal allows us to remove the noise component. However, the results of the simulation have shown that in most cases the signal is distorted. The successful results in this case can be achieved empirically by changing the thresholding parameter value. To solve this problem, we propose to process the IMFs functions which include the noise component, but not the initial signal directly. 


\section{HYBRID MODEL OF 1-D SignAl DENOISING}

Fig.3 presents the structural block chart of hybrid model of 1-D signal denoising based on the complex use of both the Huang transform and wavelet analysis. The structural block-chart of the algorithm of this process implementation is presented in Fig.4. The stages of this algorithm implementation are the following:



Fig.3. Hybrid model of 1-D signal denoising based on the complex use of both the Huang and wavelet transforms

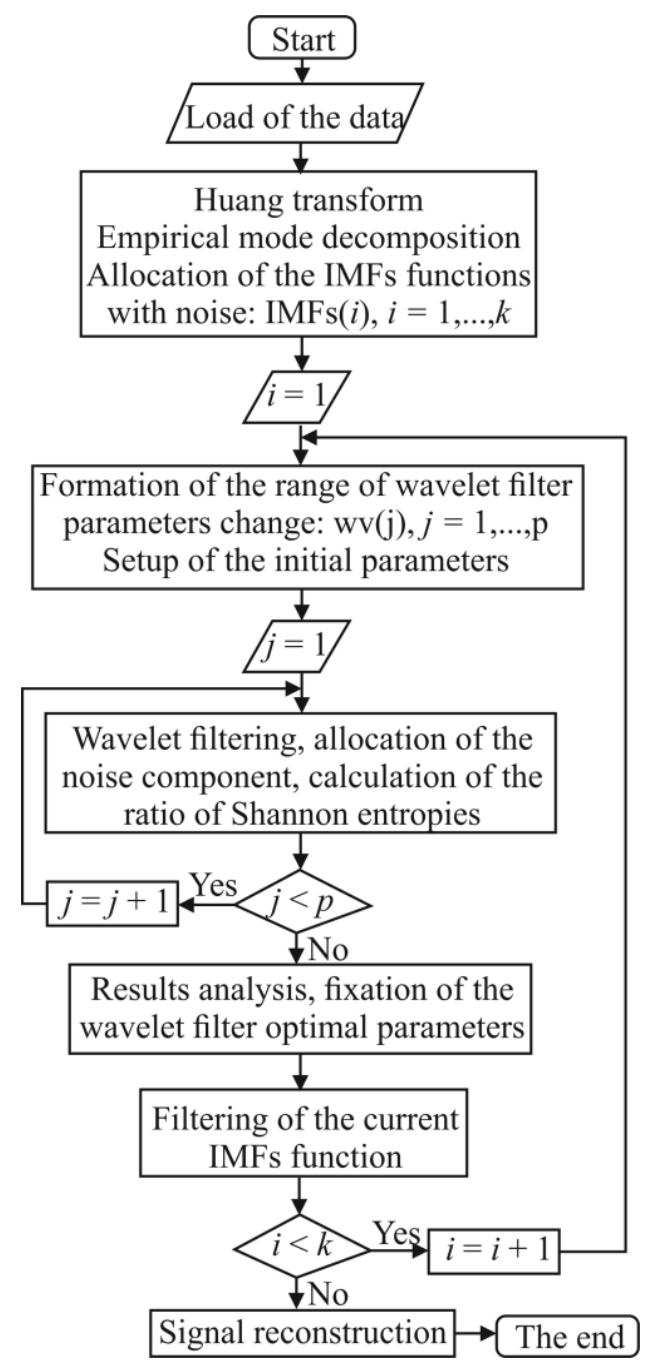

Fig.4. Block-chart of the algorithm of Huang-Wavelet filtering process implementation
Stage I. Data loading and Huang transform performing.

1. Loading of the studied signal.

2. Empirical mode decomposition by Huang transform implementation. Allocation of the IMFs functions.

3. Visualisation of the obtained functions. Results analysis. Choice of the functions with noise. Setup of the counter, which correspond to the first of the selected functions.

Stage II. Wavelet filtering of the selected IMFs functions.

4. Determination of the optimal type of wavelet.

4.1. Formation of the vector of wavelets for the appropriate mother wavelet. Setup the counter, which corresponds to the first of the wavelet in the sequence.

4.2. Setup of the wavelet decomposition level $n=4$.

4.3. Implementation of the discrete wavelet decomposition with calculation of the approximation coefficients at $n$ level and the detail coefficient at levels from 1 to $n$.

4.4. Calculation of the thresholding coefficient initial value by the formulas (4) and (5).

4.5. Soft thresholding of the detail coefficients by the formula (3).

4.6. Wavelet reconstruction of the signal with the use of both the approximation and processed detail coefficients.

4.7. Allocation of the noise component as the difference of both the initial and filtered signals.

4.8. Calculation of the Shannon entropies for both the filtered signal and allocated noise component by the formula:

$$
H=-\sum_{i=1}^{m} p_{i} \log _{2} p_{i}
$$

where $m$ is the number of the intervals, which contain the same values of the studied data; $p_{i}$ is the probability that appropriate value of the studied data belongs to the $i$-th interval. The number of the intervals is determined empirically during simulation process and this value does not change during the experiment.

4.9. Calculation of the ratio of the Shannon entropies for the filtered data and the allocated noise component by the formula (6).

4.10. If the counter is less than the maximum number of the studied wavelets in the sequence, go to the step 4.3 of this procedure. Otherwise, the analysis of the obtained results and the determination of the optimal wavelet, which correspond to the minimum value of the criterion (6).

5. Determination of the wavelet decomposition optimal level.

5.1. Setup of the range of the wavelet decomposition level change.

5.2. Repetition of the steps 4.3-4.9 of this procedure for each level of the wavelet decomposition within the given range. 
5.3. Analysis of the obtained results. Determination of the wavelet decomposition optimal level, which corresponds to the minimum value of the criterion (6).

6. Determination of the thresholding parameter optimal value for the detail coefficients processing.

6.1. Setup of both the range and step of the thresholding parameter value change: $\tau_{\min }=0.1 \tau_{0}$; $\tau_{\max }=5 \tau_{0} ; d \tau=0.02 \cdot\left(\tau_{\max }-\tau_{\min }\right)$. These values were determined empirically during simulation process.

6.2. Repetition of the steps 4.3-4.9 of this procedure for each value of the thresholding parameter within the given range.

6.3. Analysis of the obtained results. Determine the thresholding parameter optimal value which corresponds to the first achieved of the minimum value of the criterion (6).

7. Repetition of the stage II for other allocated IMFs functions with noise.

\section{Stage III. Reconstruction of the signal.}

8. Reconstruction of the signal with the use of both the processed and non-processed IMFs functions.

\section{EXPERIMENTS}

Fig.5 presents the test data, which were used during the simulation process.
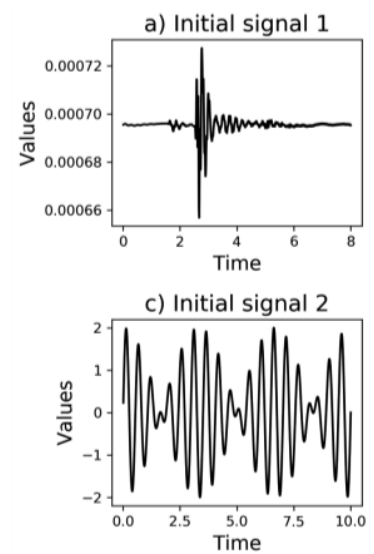
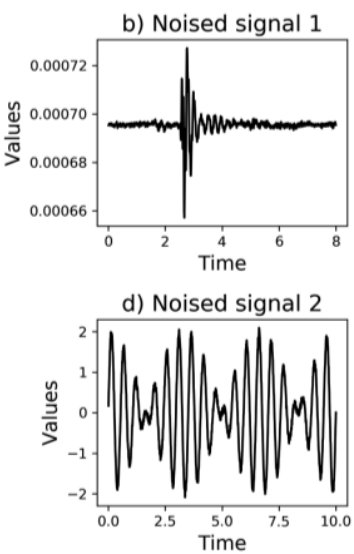

Fig.5. Test data, which were used during the simulation process: a,c) data without noise; $b, d$ ) data with noise

The first test signal contained 8 seconds of data from the 2005 TOMODEC ocean bottom seismometer network at Deception Island, South Shetland Islands, Antarctica [31]. The second test signal is the combination of two sinusoids with different frequencies $\omega_{1}$ and $\omega_{2}$ :

$$
y_{2}(t)=\sin \left(\omega_{1} \pi t\right)+\sin \left(\omega_{2} \pi t\right)
$$

The noise component was generated as the vector of random numeric values, the range of their changes corresponds to the condition:

$$
\operatorname{range}(\text { noise })=0.02 \cdot(\max (\text { signal })-\min (\text { signal }))
$$

The generated noise component was added to the appropriate signal. As the result, we have obtained the noised signals, which are shown in Fig.5(b,d). The quality of the studied signal processing was determined by calculation of the relative change of the Shannon entropy in percent calculated for both the filtered and initial signals:

$$
Q C=\frac{\mid H(\text { filt_sig })-H(\text { init_sig }) \mid}{H(\text { init_sig })} \cdot 100 \% .
$$

Less value of the criterion (8) corresponds to the better quality of the signal processing. The simulation process was performed based on the Python software use.

\section{RESULTS AND DISCUSSION}

Fig.6 and Fig.7 present the results of the Huang transform implementation for the studied signals. The first stage of the hereinbefore presented algorithm was used in this case. The analysis of the obtained results allows us to conclude that in the both cases two IMFs functions (Mode 1 and Mode 2) contain the high frequency noise component. Thus, these modes should be processed at the second step of the algorithm implementation.
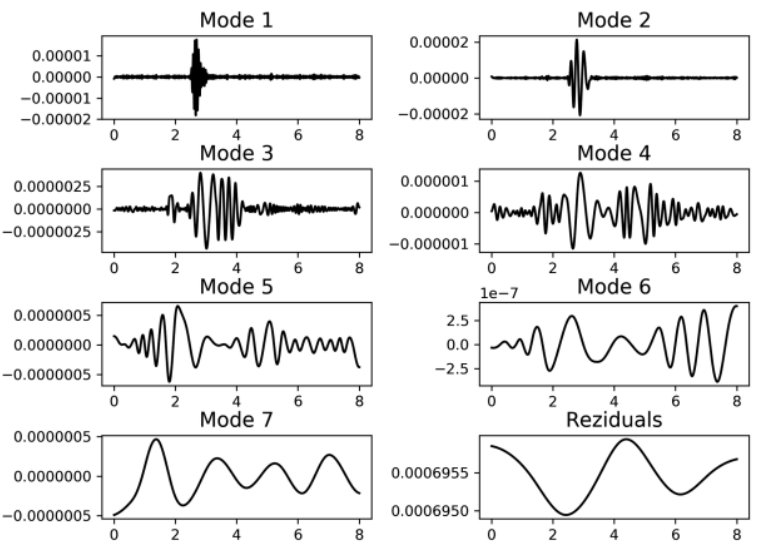

Fig.6. Results of the empirical mode decomposition for the test signal 1


Fig.7. Results of the empirical mode decomposition for the test signal 2 
Fig.8 presents the simulation results concerning determination of the optimal type of the wavelet for each of the allocated IMFs functions. The biorthogonal wavelet bior was used as the mother wavelet in this case. This choice is determined by the result of the previous simulation involving the comparisson analysis of the ortogonal and biortogonal wavelets for complex signals filtering [32].



Fig.8. Results of the simulation concerning determination of the optimal wavelet

The results of the simulation have shown that the choice of the type of the mother wavelet from orthogonal and biorthogonal wavelets in the case of the gene expression profiles filtering is not determinative. The quality of the signal filtering is determined mainly by the following parameters: type of the wavelet from the family of the mother's wavelets; level of the wavelet decomposition; value of the thresholding parameter to process the detail coefficients. The analysis of the obtained results allows us to conclude that biorthogonal wavelet bior1.1 is optimal to process IMFs_1 and IMFs_2 functions in the case of the first test signal use. In the case of the second test signal processing the wavelet biorl.5 is optimal for IMFs_1 function and the wavelet biorl.1 is optimal for IMFs_ 2 one. The values of the ratio of Shannon entropies in these cases are the minimal ones.

The results of the proposed technology implementation for purpose of the wavelet decomposition optimal level determination are presented in Fig. 9. The level of the wavelet decomposition was changed within the range from 3 to the maximum level depending on the type of the studied signal. The minimal boundary value was determined empirically.
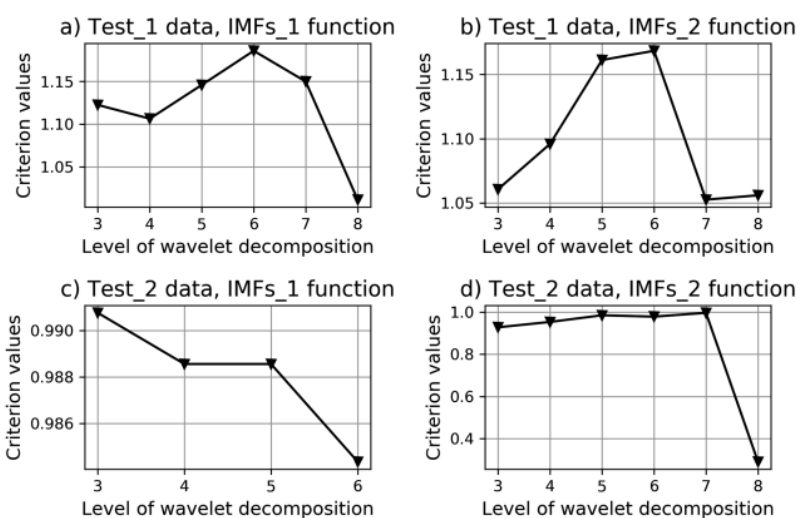

Fig.9. Charts of the Shannon entropies ratio vs the wavelet decomposition level
The results of the simulation have shown that in the case of the first test signal use the optimal level of the wavelet decomposition is 8 for the mode 1 and 7 for the mode 2 . In the case of the second test signal use the optimal levels are 6 and 8 for the mode 1 and the mode 2 respectively. These parameters were used during the following data processing.

Fig.10 presents the results of the simulation concerning determination of the thresholding coefficients optimal values to process the detail coefficients at the levels of wavelet decomposition from 1 to $n$ according to the formula (3). The range and the step of the thresholding coefficient change were determined in accordance with the step 9 of the hereinbefore described algorithm. As the results, the optimal values of the thresholding coefficients were determined for each of the allocated IMFs functions. The final stage of the data processing is the signal reconstruction with the use of both the processed and non-processed IMFs functions. The results of the wavelet filter optimal parameters determination and relative change of the Shannon entropy for the filtered and the initial signals in percent calculated by the formula (8) are presented in Table 1. Fig.11 presents the results of the test signals filtering.
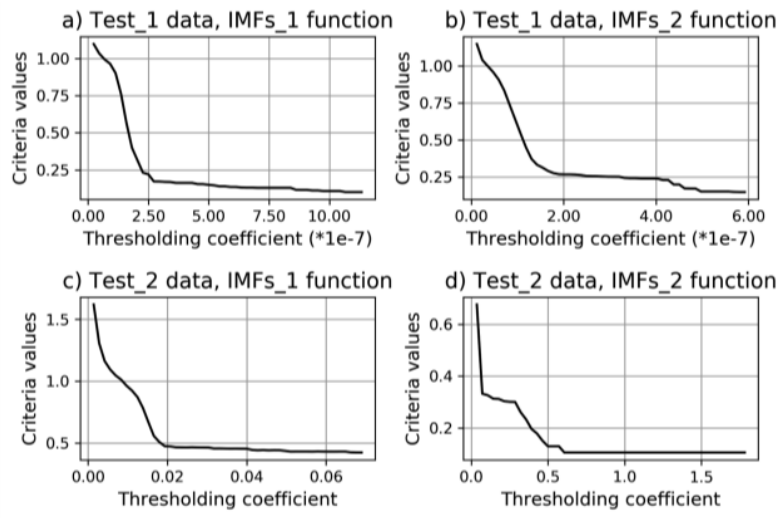

Fig.10. Charts of the Shannon entropies ratio vs the thresholding coefficient value

Table 1. Results of the simulation concerning implementation of the Huang-Wavelet adaptive filter

\begin{tabular}{|c|c|c|c|c|c|}
\hline \multirow{2}{*}{ Signal } & \multirow{2}{*}{ Function } & \multicolumn{3}{|c|}{ Parameters } & \multirow{2}{*}{ Error } \\
\cline { 3 - 5 } & & Wavelet & Level & THR & \\
\hline \multirow{2}{*}{ Test_1 } & IMFs_1 & bior1.1 & 8 & $1.07 \mathrm{e}-6$ & \multirow{2}{*}{$0.92 \%$} \\
\cline { 2 - 5 } & IMFs_2 & bior1.1 & 8 & $5.8 \mathrm{e}-7$ & \\
\hline \multirow{2}{*}{ Test_2 } & IMFs_1 & bior1.5 & 6 & 0.069 & \multirow{2}{*}{$0.42 \%$} \\
\cline { 2 - 5 } & IMFs_2 & bior1.1 & 8 & 0.607 & \\
\hline
\end{tabular}

The analysis of the obtained results allows us to conclude that the relative change of the Shannon entropies calculated for the initial and the filtered signals are less than one percent for the both test signals. This fact indicates the high effectiveness of the proposed technology. Moreover, the results of the simulation have also shown that the proposed technology is not so greatly sensitive to the thresholding coefficient value as in the case of the direct use of the wavelet analysis for the 
signal filtering. This fact can be explained in the following way. The use of the Huang transform allows us to select the noised components of the signal, which are processed at the next stage of the algorithm implementation. The components without noise are not processed. Thus, a little change of the thresholding coefficient value does not significantly influence on the signal filtering results as in the case of the direct use of the wavelet analysis for the signal denoising.


b) Filtered signal 1



d) Filtered signal 2

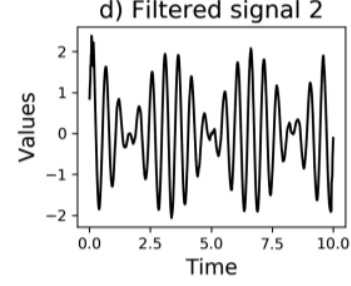

Fig.11. Results of the test signals filtering

\section{CONCLUSION}

The technology of 1-D signals filtering based on the complex use of both the Huang transform and wavelet analysis has been proposed in this paper. The implementation of this technology involves three stages. Firstly, the Huang transform is performed to decompose the signal into the components with the allocation of the components with noise. Then, the optimal parameters of wavelet filter are determined for each of the selected components. In such a way the proposed filter is adapted to the investigated signal. The wavelet filtering of the allocated components is performed as the result of this stage implementation. Finally, the reconstruction of the signal is carried out with the use of both the processed and non-processed components of the signal. The relative change of the Shannon entropy in percent calculated for both the filtered and initial test signals has been used as the quality criterion of the proposed technology implementation. The results of the simulation have shown that the values of the quality criterion are less than one percent in the both cases of the test signals use. This fact indicates the high effectiveness of the proposed technology.

The perspectives of the authors' research are the implementation of the proposed model for filtration more complex 1-D and 2-D signals.

\section{REFERENCES}

[1] Kalman, R.E. A new approach to linear filtering and prediction problems. Journal of Basic Engineering, 82(1): pp. 35-45, 1960.
[2] Wiener, N. Extrapolation, Interpolation, and Smoothing of Stationary Time Series. New York: Wiley, 1949.

[3] Staub, S., Andrä, H., Kabel, M. Fast FFT based solver for rate-dependent deformations of composites and nonwovens. International Journal of Solids and Structures, 154, pp. 33-42, 2018.

[4] Cui, L., Ma, F., Gu, Q., Cai, T. Time-Frequency Analysis of Pressure Pulsation Signal in the Chamber of SelfResonating Jet Nozzle. International Journal of Pattern Recognition and Artificial Intelligence, 32(11), art.no. 1858006, 2018.

[5] Daubechies, I. The wavelet transform, time-frequency localization and signal analysis. IEEE Transactions on Information Theory, vol. 36(5), pp. 961-1005, 1990.

[6] Coifman, R., Meyer, Y., and Wickerhauser, M. Wavelet analysis and signal processing. Wavelets and their applications, pp. 153-178, 1992.

[7] Bodyanskiy, Y., Perova, I., Vynokurova, O., Izonin, I. Adaptive wavelet diagnostic neuro-fuzzy network for biomedical tasks. 14th International Conference on Advanced Trends in Radioelectronics, Telecommunications and Computer Engineering, TCSET 2018 - Proceedings, pp. 711-715, 2018.

[8] Rouhollah Pour, H., Asgari Marnani, J., Tabatabei, A.A. A Novel Method for Crack Detection in Steel Cantilever Beam Using Wavelet Analysis by Combination Mode Shapes. International Journal of Image, Graphics and Signal Processing(IJIGSP), Vol.10, No.4, pp. 1-12, 2018.

[9] Venkata Ramana, M., Sreenivasa Reddy, E., Satayanarayana, C.H. Curvelet Transform for Efficient Static Texture Classification and Image Fusion. International Journal of Image, Graphics and Signal Processing(IJIGSP), Vol.10, No.5, pp. 64-71, 2018.

[10] Cevik, N., Cevik, T. Discrete Wavelet Transform based High Performance Face Recognition Using a Novel Statistical Approach. International Journal of Image, Graphics and Signal Processing(IJIGSP), Vol.10, No.6, pp. 1-9, 2018.

[11] Ameen, M.M., Eleyan, A. Score Fusion of SIFT \& SURF Descriptors for Face Recognition Using Wavelet Transforms, International Journal of Image, Graphics and Signal Processing(IJIGSP), Vol.9, No.10, pp. 22-28, 2017

[12] Torse, D., Desai, V., Khanai, R. Classification of EEG Signals in a Seizure Detection System Using Dual Tree Complex Wavelet Transform and Least Squares Support Vector Machine, International Journal of Image, Graphics and Signal Processing(IJIGSP), Vol.10, No.1, pp. 56-64, 2018.

[13] Hegde, G., Reddy, K.S., Shetty Ramesh, T.K. A new approach for 1-D and 2-D DWT architectures using LUT based lifting and flipping cell. AEU - International Journal of Electronics and Communications, 97, pp. 165177, 2018.

[14] Too, J., Abdullah, A.R., Mohd Saad, N., Mohd Ali, N., Musa, H. A detail study of wavelet families for EMG pattern recognition. International Journal of Electrical and Computer Engineering, 8(6), pp. 4221-4229, 2018.

[15] Huang, N., Shen, Z., Long, S., Wu, M., Shih, H., Zheng, N., Yen, N., Tung, C., Liu, H. The empirical mode decomposition and Hilbert spectrum for nonlinear and nonstationary time series analysis. Proc. Math. Phys. Eng. Sci., 454 , pp. 903-995, 1998.

[16] Huang, N.E. A review on Hilbert-Huang transform: Method and its applications to geophysical studies. Rev. Geophys. No.46, pp. 1-23, 2008.

[17] Li, W., Kuang, G., Xiong, B. Decomposition of multicomponent micro-Doppler signals based on HHT- 
AMD. Applied Sciences (Switzerland), 8(10), art. no. $1801,2018$.

[18] Soualhi, A., Medjaher, K., Zerhouni, N. Bearing health monitoring based on hilbert-huang transform, support vector machine, and regression. IEEE Transactions on Instrumentation and Measurement, 64(1), art. no. 6847199, pp. 52-62, 2015.

[19] Susanto, A., Liu, C.-H., Yamada, K., Hwang, Y.-R., Tanaka, R., Sekiya, K. Application of Hilbert-Huang transform for vibration signal analysis in end-milling. Precision Engineering, 53, pp. 263-277, 2018.

[20] Susanto, A., Liu, C.-H., Yamada, K., Hwang, Y.-R., Tanaka, R., Sekiya, K. Milling process monitoring based on vibration analysis using Hilbert-Huang transform. International Journal of Automation Technology, 12(5), pp. 688-698, 2018.

[21] Trusiak, M., Styk, A., Patorski, K. Hilbert-Huang transform based advanced Bessel fringe generation and demodulation for full-field vibration studies of specular reflection micro-objects. Optics and Lasers in Engineering, 110, pp. 100-112, 2018.

[22] Oweis, R.J., Abdulhay, E.W. Seizure classification in EEG signals utilizing Hilbert-Huang transform. BioMedical Engineering Online, 10, art. no. 38, 2011.

[23] Huang, N.E., Wu, M.-L., Qu, W., Long, S.R., Shen, S.S.P. Applications of Hilbert-Huang transform to nonstationary financial time series analysis. Applied Stochastic Models in Business and Industry, 19 (3), pp. 245-268, 2003.

[24] Yuan, H., Liu, X., Liu, Y., Bian, H., Chen, W., Wang, Y. Analysis of Acoustic Wave Frequency Spectrum Characters of Rock Mass under Blasting Damage Based on the HHT Method. Advances in Civil Engineering, art. no. $9207476,2018$.

[25] Hu, Zh., Bodyanskiy, Y., Tyshchenko, O., Boiko, O. A Neuro-Fuzzy Kohonen Network for Data Stream Possibilistic Clustering and Its Online Self-Learning Procedure. Applied Soft Computing, Vol.68, pp.710-718, 2018.

[26] Hu, Zh., Bodyanskiy, Y., Tyshchenko, O. A Deep Cascade Neural Network Based on Extended Neo-Fuzzy Neurons and its Adaptive Learning Algorithm. Proc. of 2017 IEEE First Ukraine Conference on Electrical and Computer Engineering (UKRCON), May 29 - June 2, 2017, Kyiv, Ukraine, pp.801-805.

[27] Wang, J., Wang, Y., Wang, W., Ren, S. Discrete linear canonical wavelet transform and its applications. Eurasip Journal on Advances in Signal Processing, 2018(1), art. no. 29, 2018.

[28] Babichev, S., Lytvynenko, V., Gozhyj, A., Korobchynskyi, M., Voronenko, M. A fuzzy model for gene expression profiles reducing based on the complex use of statistical criteria and shannon entropy. Proceedings of 1st International Conference on Computer Science, Engineering and Education Applications, ICCSEEA2018, Kiev, Ukraine, Vol.754, pp. 545-554, 2019.

[29] Babichev, S., Lytvynenko, V., Skvor, J., Korobchynskyi, M., Voronenko, M. Information Technology of Gene Expression Profiles Processing for Purpose of Gene Regulatory Networks Reconstruction. Proceedings of the 2018 IEEE 2nd International Conference on Data Stream Mining and Processing, DSMP 2018, art. no. 8478452, pp 336-341, 2018

[30] Babichev, S., Korobchynskyi, M., Lahodynskyi, O., Korchomnyi, O., Basanets, V., Borynskyi, V. Development of a technique for the reconstruction and validation of gene network models based on gene expression profiles. EasternEuropean Journal of Enterprise Technologies, No. 1 (4-91), pp. 19-32, 2018

[31] Bowman, D.C., Wilcock, W.S.D. Unusual signals recorded by ocean bottom seismometers in the flooded caldera of Deception Island volcano: Volcanic gases or biological activity. Antarctic Science, 26(3), pp. 267-275, 2014.

[32] Babichev S. Technology of wavelet-filtration of the gene expression profiles in order to remove the background noise. Control Systems and Computers, No. 5, pp. 25-42, 2017.

\section{Authors' Profiles}

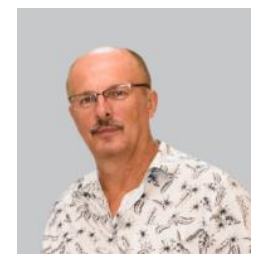

Sergii Babichev graduated (MSc.) from Kherson State Pedagogical Institute in 1984. He got his PhD in 2003 and DrSc in 2018.

$\mathrm{He}$ works currently as Associate Professor of both at the Department of Informatics at Jan Evangelista Purkyně University in Ustí nad Labem, Czech Republic and the Department of Information Technologies at IT Step University, Lviv, Ukraine. He has about 100 scientific publications. His research interests are: data mining of complex data; bioinformatics; gene expression profiles processing; gene regulatory networks reconstruction and simulation.

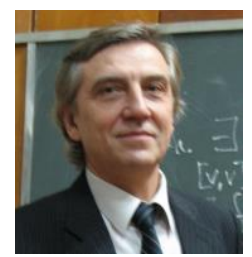

Alexandr Mikhalyov graduated from Dnipropetrovsk State University in 1976. He got PhD in 1980 and $\mathrm{DrSc}$ in 1993, full professor in 2001.

He works currently as professor, head of information technologies and systems department National metallurgical academy of Ukraine. He has more 300 scientific publications. His research interests are in field of the theory of adaptive identification and neuro-fuzzy control of nonlinear dynamical systems, network technologies; mathematical simulation of technological processes and data mining, the fractal material science and applied synergetic.

How to cite this paper: Sergii Babichev, Oleksandr Mikhalyov, "A Hybrid Model of 1-D Signal Adaptive Filter Based on the Complex Use of Huang Transform and Wavelet Analysis", International Journal of Intelligent Systems and Applications(IJISA), Vol.11, No.2, pp.1-8, 2019. DOI: 10.5815/ijisa.2019.02.01 